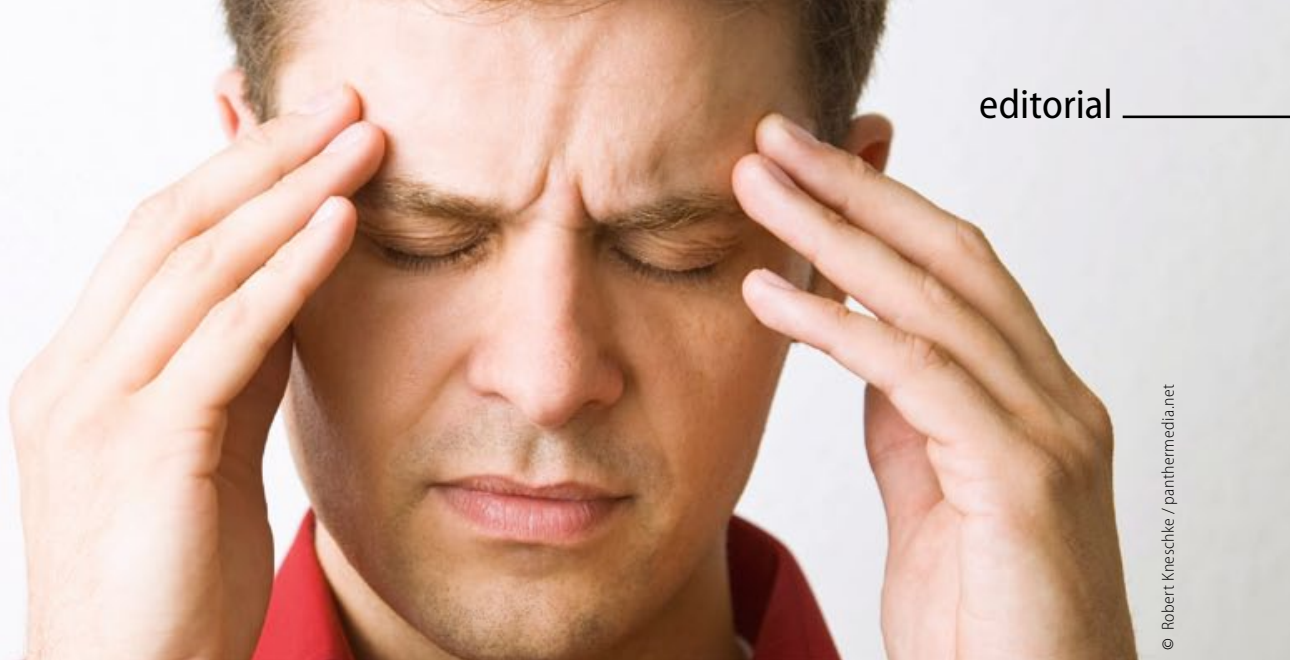

Migräne-Chirurgie

\title{
Körperverletzung unter erheblichem finanziellen Aufwand
}

Chronische Krankheiten, die schwer zu behandeln sind wie die Migräne, führen häufig dazu, dass Patienten frustriert sind und zu Außenseitermethoden greifen. Solange dies Therapien wie Homöopathie und Akupunktur sind, kann zumindest kein weitreichender Schaden angerichtet werden [1]. Ganz anders ist die Situation, wenn es sich um eingreifende, insbesondere operative Therapien handelt.

Vor einigen Jahren hatte ein plastischer Chirurg in den USA beobachtet, dass einige seiner Patientinnen im Alter von Mitte 50 nach kosmetischer Entfernung des M. corrugator über eine Besserung ihrer Migräne berichteten. Er führte dann eine Reihe offener Studien durch, bei denen er entweder den M. corrugator entfernte oder eine sogenannte Neurolyse im Bereich des M. temporalis oder des N. occipitalis major vornahm [2]. Er und seine Mitarbeiter führten dann sogar eine randomisierte Studie durch und belegten vermeintlich, dass diese operative einer konservativen Therapie überlegen sei [3]. Die Studie hatte allerdings alle methodischen Mängel, die überhaupt nur möglich sind [4]. Besonders eindrucksvoll war, dass mehr als die Hälfte der Operierten von der Migräne komplett „geheilt“ waren.

Bis zu meinem letzten Besuch in den USA, der mit der Aufgabe verbunden war, auf dem Internationalen KopfschmerzKongress Dr. Bahman Guyuron in einer Pro- und Kontra-Diskussion zu konfrontieren, war mir die praktische Konsequenz seiner Publikationen nicht in vollem Umfang bewusst. Es gibt in den USA inzwischen mehr als 30 Zentren für plastische Chirurgie, die diese Methode anbieten. Einige dieser Zentren versprechen eine über $90 \%$ ige Erfolgsrate bei der chirurgischen Therapie der Migräne. Leider ist diese Welle auch nach Deutschland übergeschwappt und es gibt in der Zwischenzeit im Internet eine ganze Reihe von sogenannten Migräne-Chirurgie-Zentren, die diese operative Therapie anbieten.

Es ist eine besondere Aufgabe von klinisch tätigen Neurologen, Kopfschmerz- und Migräne-Patienten darauf aufmerksam zu machen, dass es sich hier nicht um eine wirksame Therapie, sondern um Körperverletzung unter erheblichem finanziellen Aufwand handelt. Im Gegensatz zu den USA sind in Deutsch-

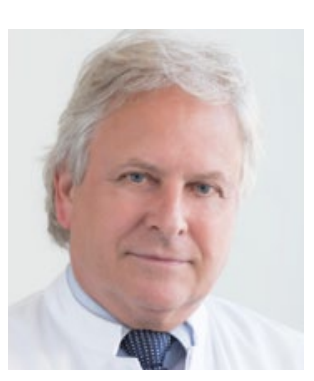

\author{
Prof. Dr. med. Hans-Christoph \\ Diener, Essen \\ Direktor der Klinik für Neurologie, \\ Universitätsklinikum Essen \\ E-Mail:h.diener@uni-essen.de
}

land erfreulicherweise die meisten Krankenkassen nicht bereit, diesen Eingriff zu bezahlen. Meine US-amerikanischen Kolleginnen und Kollegen haben eine ganze Reihe von Patienten mit anschließender therapierefraktärer Anaesthesia dolorosa beobachtet.

Wir müssen als Neurologen aber nicht nur gegenüber chirurgisch tätigen plastischen Chirurgen kritisch sein. Auch innerhalb unserer eigenen Berufsgruppe macht sich im Moment eine unangemessene Euphorie bezüglich operativer Therapie beim chronischen Cluster-Kopfschmerz und bei der chronischen Migräne breit [5-9]. Und dies obschon die bisher durchgeführten randomisierten Studien mit wenigen Ausnahmen eine klare Überlegenheit gegenüber einer Scheinstimulation bei zugegebenermaßen kleinen Patientenzahlen nicht belegen konnten. Daher sollten Verfahren, wie die bilaterale Stimulation des N. occipitalis oder die externe Stimulation des Ganglion sphenopalatinum nur im Rahmen von randomisierten Studien und prospektiven Registern an Zentren durchgeführt werden, die bereits Erfahrung mit diesem operativen Eingriff und insbesondere mit den daraus resultierenden Komplikationen haben.

Hans Christoph Diener 